\title{
Virtual Wireless and Mobile Communication Laboratory
}

\author{
Ahmad Nassar, Motaz Mohammed, Ali Elrashidi", Khaled Elleithy \\ Department of Computer Science and Engineering, University of Bridgeport, CT 06604, USA
}

\begin{abstract}
Creating a virtual laboratory for distance learning courses has become recently so important recently for engineering education. In this paper, we provide a full description for a remote access technique used in a wireless and mobile communication laboratory. Hence, the student will be able to perform experiments online and controlling and watching the devices by accessing a camera already built in the laboratory. Signal generator, spectrum analyzer and field-fox devices are used in the virtual laboratory.
\end{abstract}

Keywords Virtual Laboratory, Remote Access, Distances Learning, Signal Generator, Spectrum Analyzer, Field-Fox Device

\section{Introduction}

Studying a course via the internet means that a student can interact with their teacher via email and video conferences. This educational method requires a high degree of maturity on the student behalf, therefore it is more suitable for graduate students and senior undergraduates who are taking courses that require practical laboratory practices[1].

With the beginning of the internet for more than two decades ago, distance education has been introduced and expanded through universities and schools. One of the most important factors of distance education was the development of "distance experimentation". This kind of education focuses mainly on practical aspects of instructional systems, to give students theory and practices and also to help teachers and professors who are not always physically on site. There are a lot of difficulties in this area and there has not been so much work done in this kind of educational field until lately.

Remote access experimenting has become so important recently for engineering education because it made it easier to provide more lab hours and also make efficient use of equipments and providing convenience for offsite students[2].

Since 1994, Engineers and others have established the possibility of making distance experimentation easy and accessible by student for their practical experiments and using the internet as a way to learn. One of the earliest experiments and approaches to distance experimenting was robot control and some circuit fundamental experiments[3].

As universities expand distance education programs, they offer educational opportunities in different market sectors

* Corresponding author:

alirashedy@gmail.com (Ali Elrashidi)

Published online at http://journal.sapub.org/edu

Copyright (C) 2012 Scientific \& Academic Publishing. All Rights Reserved than they usually operate. Universities may use this new technology to attract students in nontraditional markets in which could result in competition or cooperation between universities. Universities may also share access to one internet based access of this new technology.

For distance experiment, it is really important to provide very high quality lab experience to the offsite students. Early remote access lab implementations were proofs of the practicability of this new Technology which has absorbed much of the cost of providing lab services to a limited set of students.

Many public universities have faced budget cuts over the past few years, the support needed for computing systems and labs was usually provided by graduate teaching assistants, who change from one semester to another. decisions must be made on how this new technology should be delivered according to who should provide the laboratory services, what kind of support, what are the available lab hours needed, and finally what kind of infrastructure is required for distance experimenting[4].

\section{Related Work}

One of the newest and increasingly popular technologies is remote lab access. With the world wide spread of the Internet and multiple applications that come with it, remote access technology has become a high demand technology[5].

An electronic remote access FPGA laboratory was developed by Hashemian and Riddley[5]. This system was intended for the use of the FPGA and Xilinx's Spartan-3E Starter Kit that are used in digital logic design laboratory. The remote desktop application provided by windows XP is used to connect a user with the FPGA board, LabView, and hardware such as an integrated webcam, GPIO and data acquisition. GPIO and the integrated webcam then connect to the switches, LEDs, push buttons and the rest of the 
hardware on the FPGA board itself. A benefit of using this methodology is the inexpensive setup cost that comes with the FPGA Spartan-3E Starter kit. However, using windows XP remote desktop kit slows down the system.

NetLab design started in the University of South Australia and was developed in order to overcome the most common problems regarding remote access laboratory. The architecture of NetLab is similar to previous work, but in addition it includes a selection of materials and equipment incorporated in the design and it allows collaboration between multiple users. The end result of the Netlab research was that standard working labs outperform remote access labs.

To evaluate the proficiency of conventional labs simulated labs, and remote $\log$ in labs, Corter et.al.[6] proposed a model to compare the results of each[7]. The main focus of the experiments was the motion and activity of apparatus such as cameras, microphones, connection and gears. Six experiments were executed; three of them were hands-on in person and the other three were remote lab access. The results that were closely looked at by the conductors were preferences and overall satisfaction.

Learning ability and their style of thinking were the main characteristics related to students preferred remote access laboratories[7]. The final results showed that the least preferable criteria by students included team work, pre lab experiments, final reports and not surprisingly the attendance of the lab. The degree of how much a student learned from the experiments was tested by professors on the midterms and final exams.

Remote laboratory access was also used for an optical circuit class[8]. The purpose was to educate the students about the basics and concepts before moving on to pre laboratory work[9]. Pre-laboratories had on-line material that consists of an introduction video, results, and the step by step laboratory procedures. The learning outcome was measured and the teaching methods were assessed. Students were required to connect to a LabView web Server using a specific client. Afterwards, they were required to perform three experiments.

The characterization of optical source circuits, the attenuation of optical fiber links and connectors, and practical skills obtained by students from performing remote labs. The success was measured based upon how successful each student was in conducting these three experiments. Afterwards, a student survey was taken to gauge their opinion.

The ILab technology was first developed at the Massachusetts Institute of Technology (MIT)[10]. A remote access biology laboratory was prepared by Elawady et.al. using the ILab. The benefits of performing a general biology lab remotely according to Elawady et.al. were that resources and costs are shared, which leads to a more efficient and productive work environment. The work by Elawady et.al. inspired other researches to start looking into remote access laboratory in their fields and acknowledging some of the benefits that come along with it.

One field that is being looked into is FPGA because the boards themselves can be manipulated and are flexible.
There is not that much work out there that involves remote accessing the FPGA board, however, more research is being carried out and the interest is growing[11].

\section{Proposed Laboratory Overview}

In Figure 1, the overall proposed laboratory is illustrated. The student will be able to access the laboratory server via Internet and by using username and password assigned by a laboratory monitor student can access the laboratory devices. By using an integrated camera in the lab, the student will be able to check any experiment connections or electronic circuit connections.

Signal generator and spectrum analyzer are used to generate and analyze signals for most of the experiment in the designed laboratory. Field-fox device is used as either network analyzer mode or signal generator mode. Field-fox is a very efficient device in most of experiments in the laboratory.

A camera is connected to a personal computer very close to the devices and mainly controlled by a laboratory monitor person. A student is able to access the camera via internet connection to watch the devices setup in the laboratory.

A lab monitor will be responsible for giving a username and password to each student registered in course, do the setup for experiment each week and set a time slot for each student, so the student can access the devices in his/her own time slot during the week.

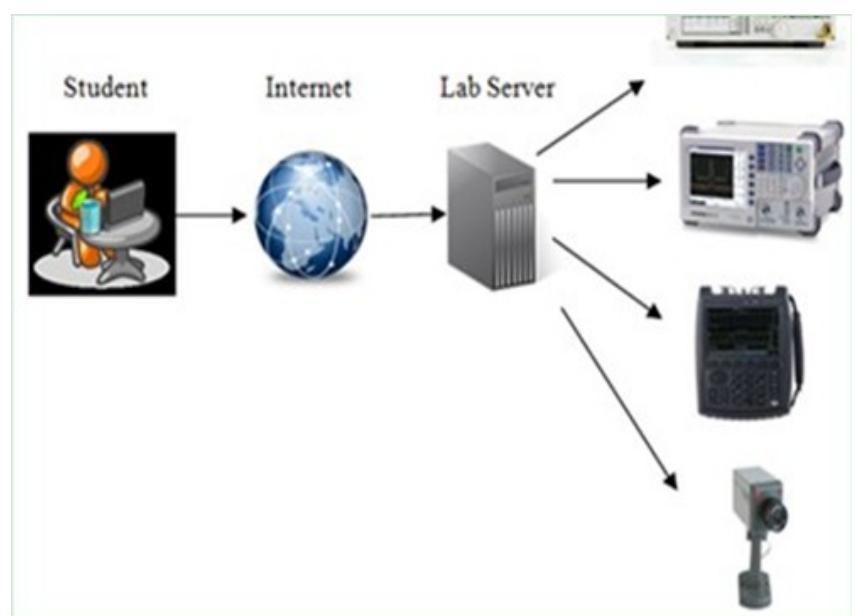

Figure 1. Overall system overview for accessing the virtual laboratory

Any experiment can be accessed by only one student at a time, and the student can chat immediately with the laboratory monitor if the student has any questions or concerns.

\section{Remote Access to the X-Series Signal Analyzer and the MXG Signal Generator}

There are two instruments that the virtual laboratory can use remotely logged into; the X-Series signal analyzer and 
the MXG signal generator. For the X-series signal analyzer, the remote login in can be achieved via the local area network (LAN) or using an embedded web server. On the other hand, for the MXG generator, remote login can only take place through a web-enabled page because it is not running on any operating system.

\subsection{X-Series Signal Analyzer}

\section{LAN configuration}

The initial step is to set up the instrument and prepare it to remotely connect to a computer. An administrator level access to the instrument is required. The following steps are executed:

1. First, go to the control panel on the instrument and double click on system.

2. Next, click on the remote tab and ignore the warning message that pops up.

3. Then check the box to allow users to remotely use this computer and click the select remote users and then add.

4. Follow the on-screen instructions and now the Signal analyzer can be accessed.

Now that the instrument is set up, the next step is to set up the remote host computer. First, the remote desktop connectivity software is needed. If windows XP or any later operating system versions are being used, it automatically comes installed with it. If using an earlier windows version, the software must be installed from a CD. The next step is to get the instrument's name by pressing system, show, system on the front panel and the name will appear.

To start the remote desktop session, from the remote computer, go to start, all programs, accessories, communication, remote desktop connection and then enter the name of the instrument you have obtained and click connect. Enter the login and password and click ok which will bring up the remote desktop connection screen. Ensure that the login name, password, and computer name are all correct and then under local resources, check the disk drives and printer boxes to enable transfer between the instrument and the computer, and click connect. This will make the remote desktop connection and as Figure 2 display.

\section{a) Internet connection}

Using an embedded Web server is valuable when you cannot log onto the instrument's account. You can wirelessly connect to the instrument without needing to log the current user off. Also, this function only works with Internet explorer. The first step is to enter the URL consisting of the hostname or IP address, which will take the user to the welcome page. On the welcome page, there are tabs that enable the user to change any configuration on information about the instrument, and it is easy to change or modify them. Next, click control and enter the password (which is "Agilent" by default). This will bring up instrument's control web page, and then by clicking display front panel keys, a virtual keyboard will appear identical to the figures from the desktop remote login method.

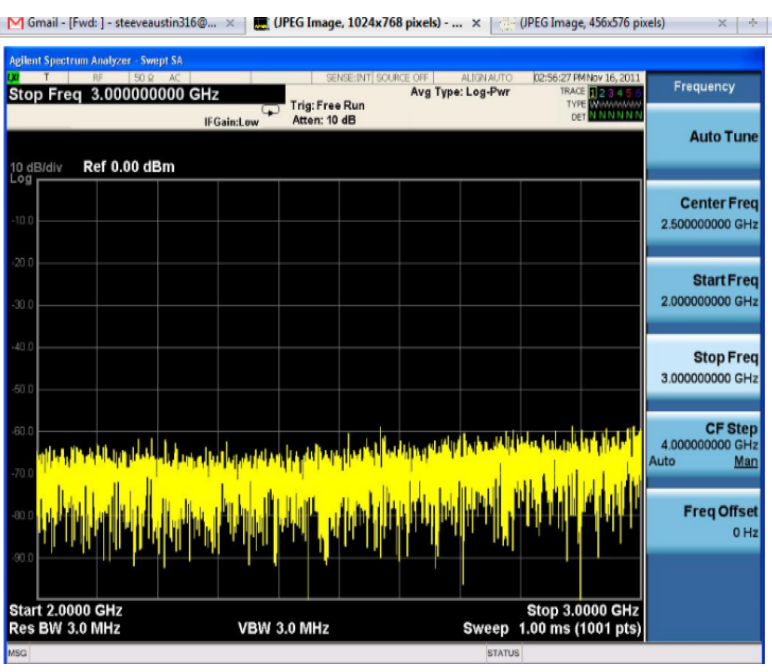

Figure 2. spectrum analyzer results

If you navigate to utility, virtual front panel, a panel identical to the instrument's front panel will appear as shown in Figures 3 and 4.

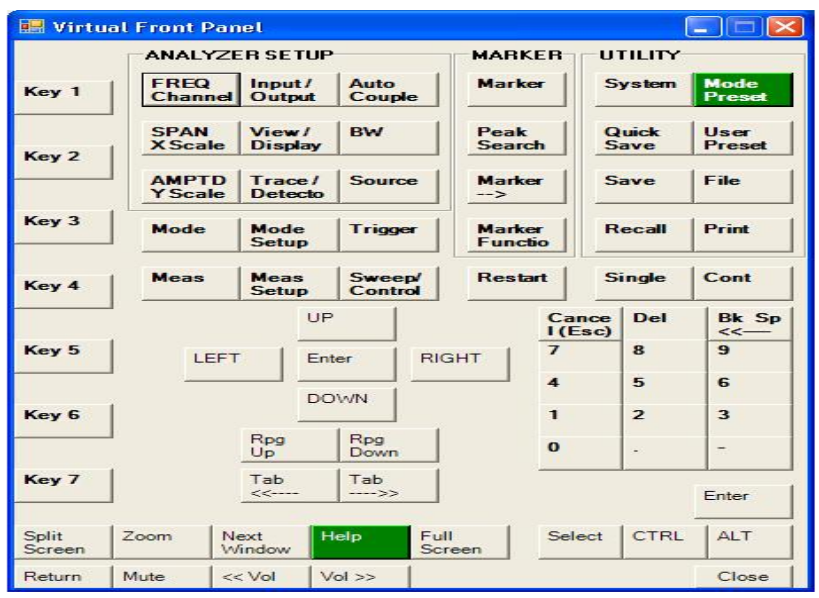

Figure 3. first spectrum analyzer virtual panel

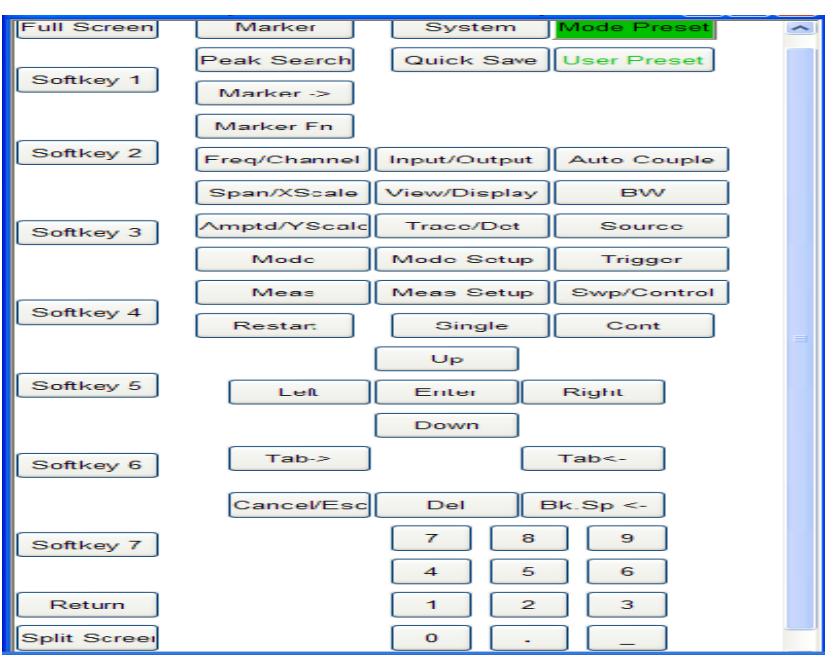

Figure 4. second spectrum analyzer virtual panel

\subsection{MXG Signal Generator}

The only way to remotely access the MXG signal generator is through a web-enabled client. To do so, enter the 
URL starting with a- then follow by instrument's model name and the last five digits of the instrument's serial number. This will take you to the homepage. By clicking signal generator web control from the menu, the virtual front panel of the MXG generator will appear. If asked for a password, by default it is set to "Agilent". The screen shot below shows the virtual front panel, Figure 5.

Now that the virtual front panel has been accessed, it is advised to adjust the brightness of it and turn the screen saver off. Also, the panel can be easily configured to match the specifications of the used operating system. Generating a signal can be easily done by manipulating the frequency on the panel as desired.

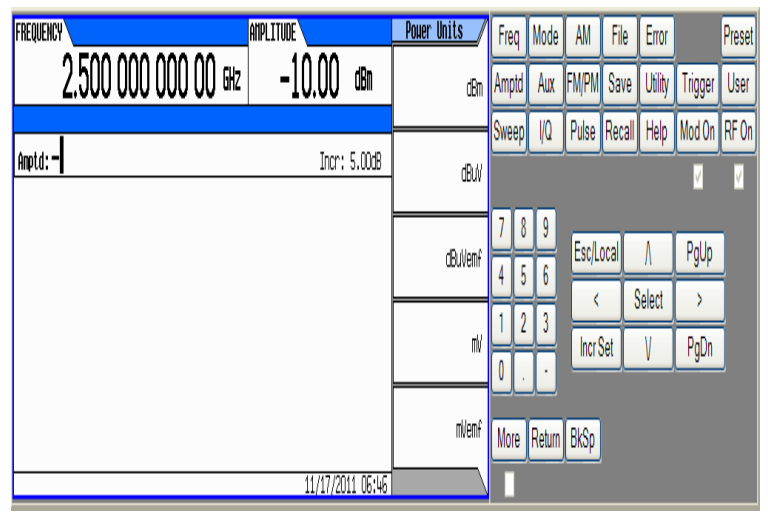

Figure 5. signal generator panel

\subsection{Field-Fox Analyzer}

The Field fox analyzer is a portable instrument which is used on the field to analyze signals and networks. The field Fox analyzer instrument has a LAN port but however it wasn't designed for to remotely login on the instrument because of its portability.

However we have been able to figure out how to remote access to the device using specific software (remote display control for windows $\mathrm{CE}$ ) although the LAN port on the device was designed for and used for sending and receiving data files only.

The Remote display control for windows CE is simple software that is basically used for remote accessing mobile devices and since the field fox analyzer is in the same category we were able to remote access the device using that software[12].

Details on how to remotely access the Field Fox analyzer is shown next. First we need to set up the instrument and connect to the Internet via an LAN cable. After that we start setting up the network for the device by assigning it an IP address which will be used to login on the device we do that by going to "system preferences" and then we choose "LAN". All network settings will show up so we scroll down to "apply setting" and then we choose "power up", the device will present the LAN settings and give the device an IP address.

Once the instrument is set and ready, we use any computer and launch the "remote display control "application as in Figure 6.

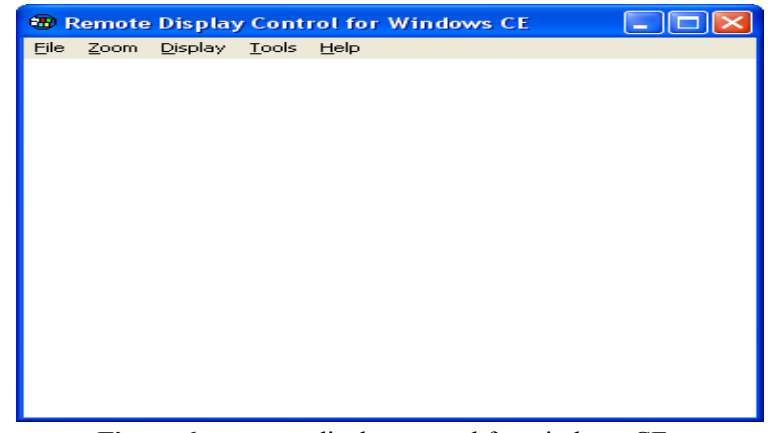

Figure 6. remote display control for windows CE

Next we click on "file" and then click on "connect". A pop window will show up asking to enter the IP address of the mobile device we wish to connect to as in Figure 7.

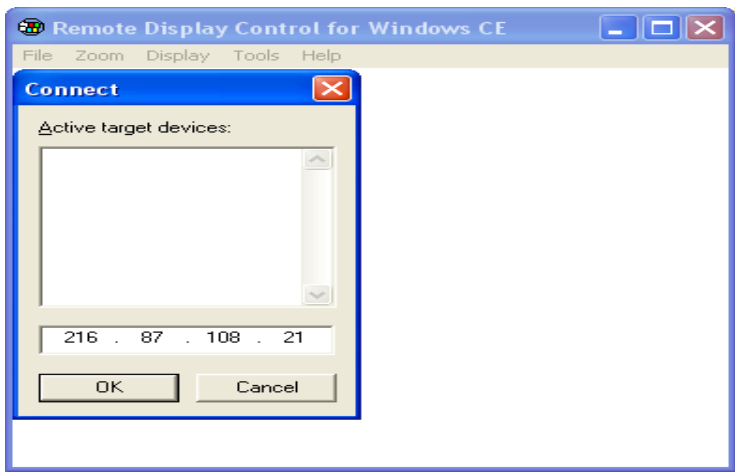

Figure 7. accessing the device via its IP address

Once you click "OK", the application will have full remote access to the device as in Figure 8.

As for controlling the device the mouse of the computer should be able to control the device and also for some special keys such as numbers they can be used from the regular keyboard of the Computer.

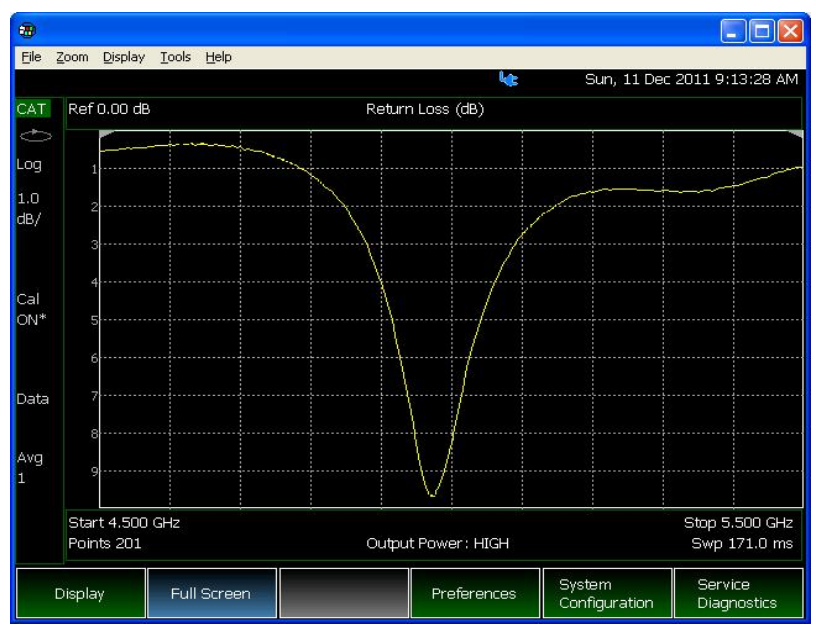

Figure 8. field-fox display

\section{Accessing an Experiment}

A student will be able to access the devices after listening to a short demo, posted in the virtual laboratory website, 
describing the accessing procedures. The laboratory monitor is responsible for electrical connections for the experiments in the laboratory.

\section{Measuring the Performance of A Microstrip Antenna}

In this experiment, the student is required to measure the performance of a printed microstrip antenna. Those parameters are voltage standing wave ratio, return loss and input impedance for a flat microstrip printed antenna and the measurements should be done in two parts as shown bellow.

Figure 9, appears when the student access the camera. In Figure 9, a flat microstrip antenna connected to the signal generator and the antenna under test is connected to the spectrum analyzer. In the first part of the experiment, student need to set the frequency of the signal generator to be $2.5 \mathrm{GHz}$ from the signal generator panel and set the amplitude to be $-10 \mathrm{dBm}$.

The measurements will be shown in the spectrum analyzer screen. It can be controlled using analyzer virtual panel, as introduced in the previous section. The image of the spectrum analyzer is shown in Figure 10. A peak at 2.5 $\mathrm{GHz}$ is obtained on the spectrum analyzer panel, and the peak value can be measured by using the marker from the control panel of the analyzer.

In the second part, the student is asked to measure some parameters to get the performance of the antenna. Those parameters are voltage standing wave ratio, return loss and input impedance of the antenna. Figure 11 shows the field-fox device that measures the return loss of the connected antenna. The student is able to change the starting and ending frequencies to clearly show the performance parameter of the antenna.

Voltage standing wave ratio and input impedance of the designed antenna can also measured using a field-fox device.

Students can save the output data in a Matlab format in their personal computer and analyze the data using Matlab program. The output data is shown in Figure 12 using Matlab program.

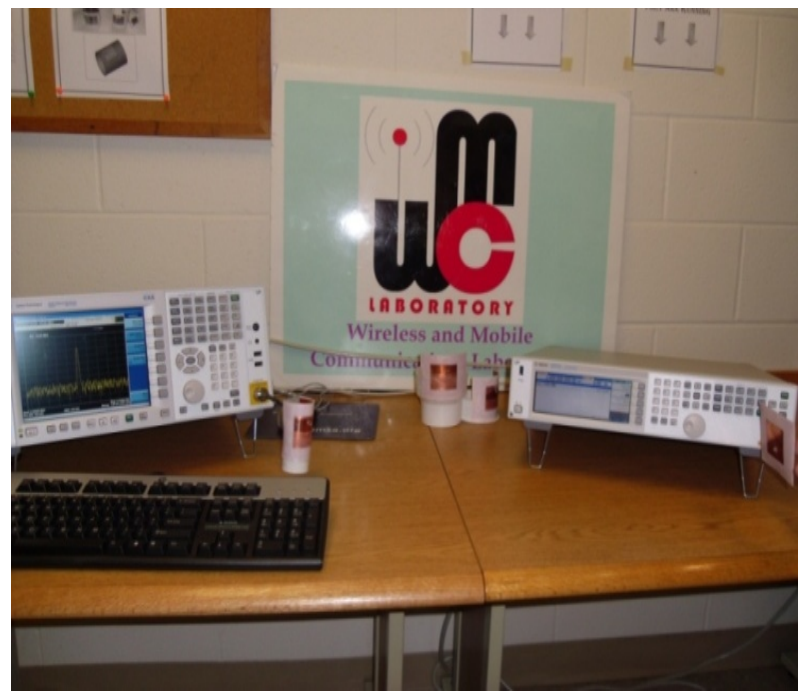

Figure 9. performance measurement of a microstrip printed antenna

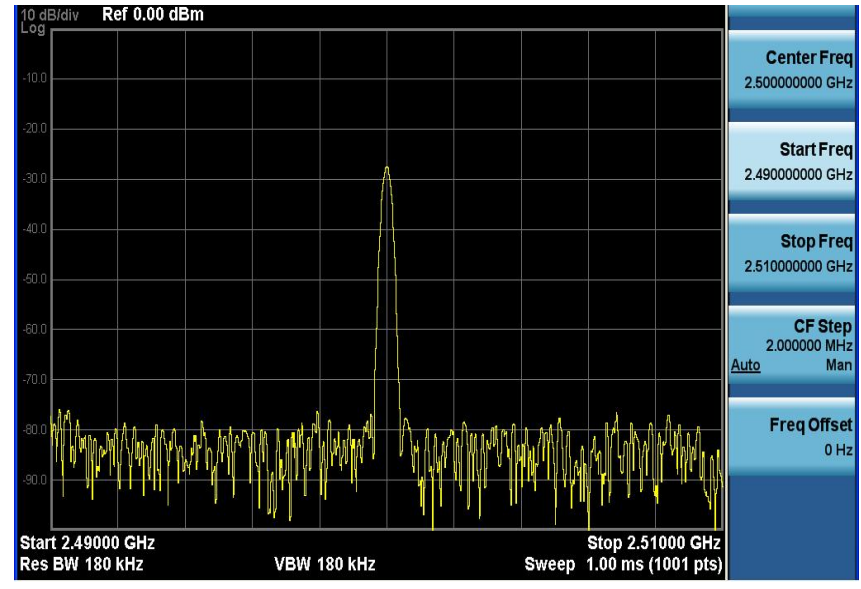

Figure 10. screen shut of the spectrum analyzer

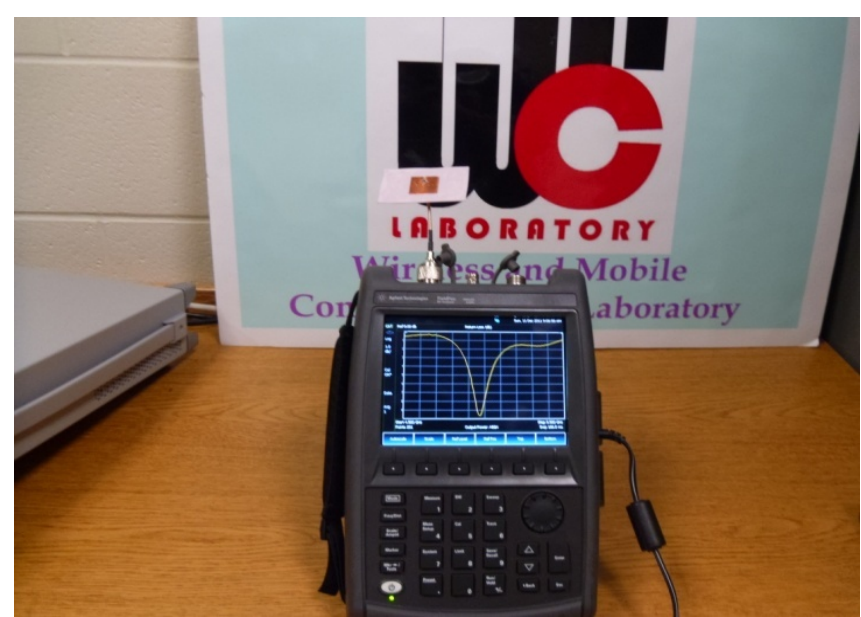

Figure 11. voltage standing wave ratio for the designed antenna shown in field-fox device

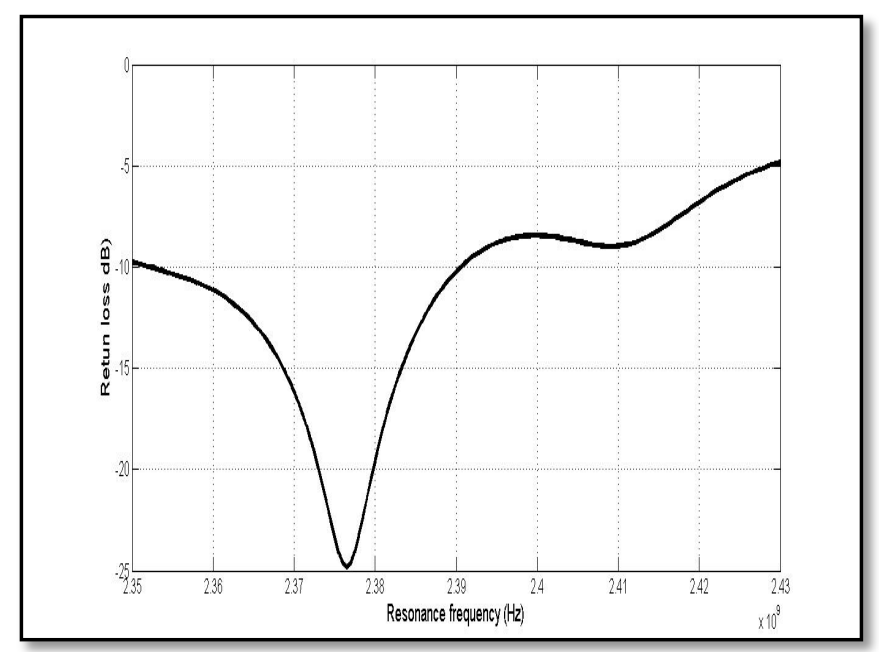

Figure 12. return loss as shown in Matlab program

\section{Conclusions}

In this paper, we introduce the mobile and wireless virtual laboratory that is designed to be one of the distance learning courses. A full description of the remote access technique used for the wireless and mobile communication laboratory 
using a fixed camera in the laboratory is introduced. Accessing the signal generator, spectrum analyzer and field-fox devices then controlling these devices using remote access technique are introduced.

An implementation of a real experiment in the wireless and mobile communication laboratory for a microstrip antenna is given in this paper. Return loss, voltage standing wave ratio and input impedance for a microstrip printed antenna are measured and saved in the personal computer and processed in Matlab software program.

\section{REFERENCES}

[1] Whelan, "Remote Access to Continue Engineering Education - RACeE," IEE Engineering Science and Educati -onal Journal, 1997

[2] Reza, "FPGA E-Lab, A Techinique to Remote Access a Laboratory to Design and Test," IEEE International Conference on Microelectronic System Education, San Diego, ca., 3-4 june, 2007

[3] Aktan, Bohus, Crowl and Shor, "Distance Learning Applied to Control Engineering Laboratories," IEEE Trans. Education, 39 (3), pp. 320-326, Aug. 1996

[4] shor, "Remote-Access Engineering Educational Laboratories," at Oregon State University , Corvallis, 2000
[5] Hashemian and Riddley, "FPGA E-lab, A Technique to Remote Access a Laboratory to Design and Test," IEEE International Conference on Microelectronic Systems Education, MSE07, June 2007

[6] http://vipmeister.com/dl/netlab/netlab.html

[7] Corter, Nickerson, Esche, Chassapis, and Ma,"Constructing Reality: A study of Remote, Hands-on, and Simulated Laboratories," ACM Transaction, Computer Human Interaction, 2007

[8] Nedic, Machotka, and Nafalski, "Remote Laboratories Versus Virtual and Real Laboratories," Frontiers in Education (FIE 2003) $33^{\text {rd }}$ Annual, 2003

[9] Gurkan, Mickelson, and Benhaddou,"Remote Laboratories for Optical Circuits," IEEE Transactions on Education, Feb. 2008

[10] Elawady, Yasser, and Tolba "A General Framework For Remote Laboratory Access: A Standarization Point of View," IEEE Faculty of Computers and Information Technology, 2011

[11] Elawady, Yasser, and Tolba "Analysis, Design and Implementation of a General Framework for Remote Lab," International Journal of Computer Applications January 2011

[12] http://na.tm.agilent.com/fieldfox/download_files/cerhost60.z ip 